L. da Silva, M. Spite, J. R. de Medeiros, eds.

\title{
The Deuterium Abundance In The Galactic Center 50 km/s Molecular Cloud: Evidence For A Cosmological Origin Of $\mathbf{D}$
}

\author{
D. A. Lubowich
}

Dept. of Physics, Hofstra U., Hempstead, NY \& AIP, Melville, NY

Jay M. Pasachoff and Robert P. Galloway

Dept. of Astronomy, Williams College, Williamstown, MA

Thomas J. Balonek and Christy Tremonti

Dept. of Physics, Colgate University, Hamilton, NY

Tom Millar and Helen Roberts

Dept. of Physics, Univ. Manchester Institute of Technology, Manchester, UK

\begin{abstract}
We confirm that deuterium exists in the Galactic Center (GC) and estimate that $\mathrm{D} / \mathrm{H}=3 \times 10^{-6}$ using a new 5192-chemical reaction model. This is the lowest $\mathrm{D} / \mathrm{H}$ ratio observed in the Galaxy, five times lower than the local ISM $\mathrm{D} / \mathrm{H}=1.5 \times 10^{-5}$ but $10^{6} \times$ larger than $\mathrm{D} / \mathrm{H}$ predicted by GC models. We detected DCN in the GC Sgr A $50 \mathrm{~km} / \mathrm{s}$ molecular cloud located $10 \mathrm{pc}$ from the GC with the NRAO $12 \mathrm{~m}$ telescope and obtained $T_{R}^{*}=0.061 \pm 0.007 \mathrm{~K}$ and $0.04 \pm 0.02 \mathrm{~K}$ for the $J=1-0$ and 2-1 lines. The most likely source of the GC D is continuous injection from the infall of primordial matter with $\mathrm{D} / \mathrm{H}=$ $5 \times 10^{-5}$ with the $\mathrm{D} / \mathrm{H}$ determined by astration and mixing. Thus there are no significant Galactic sources of $\mathrm{D}$ and no recent quasar or AGN activity in the GC. This primordial D/H implies that the baryon density is less than the density necessary to close the Universe; most of the baryons are in dark matter; and there are fewer than four $\nu$ families.
\end{abstract}

\section{Introduction and observations}

Because $\mathrm{D}$ is not produced in stars, the abundance of $\mathrm{D}$ will decrease with time unless there are any additional sources of $\mathrm{D}$. The $\mathrm{D} / \mathrm{H}$ ratio is an important prediction of big-bang nucleosynthesis(Schramm \& Turner, 1998) because D/H depends on the $\mathrm{T}$ and baryonic density during the first 1000 seconds and might determine if the density is sufficient to close the universe. Recent observations of D including QSO absorption spectra are reviewed by Vidal-Madjar (1999).

The GC is the most active and heavily processed region of the Galaxy and the Sgr A $50 \mathrm{~km} / \mathrm{s}$ cloud (M-0.02-0.07), $10 \mathrm{pc}$ from the GC, is related to the 
GC activity and is the best place to search for Galactic D. If D is produced by any stellar or Galactic process, then it should be more abundant in the GC and there should be a corresponding gradient in the D abundance (Pasachoff \& Vidal-Madjar 1989). Chemical models of the GC with no sources of D predict $\mathrm{D} / \mathrm{H}=5 \times 10^{-12}$ at $12 \mathrm{Gyr}$ (Audouze et al. 1976).

We used the NRAO 12-m telescope during May 16-18, 1993, and June 29, 1993, and observed the DCN $\mathrm{J}=1-0$ and $\mathrm{J}=2-1$ lines at $72.404 \mathrm{GHz}$ and 144.83 $\mathrm{GHz}$ in total-power mode using position switching with the 3 -mm and 2-mm SIS receivers centered at $\alpha=17^{h} 42^{m} 42^{s} \delta=-28^{\circ} 58^{\prime} 00^{\prime \prime}$ (the peak CS J=7-6 and $\mathrm{J}=5$-4 emission; Serabyn, Lacy, \& Achtermann 1992) insuring that we observed the densest part of this cloud $\left(n=10^{6} \mathrm{~cm}^{-3}\right.$ We analyzed the data with the UNIPOPS program. We detected both the $\mathrm{J}=1-0$ and $\mathrm{J}=2-1$ lines of DCN and obtained $\mathrm{T}_{R}^{*}=0.061 \pm 0.007 \mathrm{~K}$ and $\mathrm{T}_{R}^{*}=0.042 \pm \mathrm{K}+/-0.02 \mathrm{~K}$, respectively, where $\mathrm{T}_{R}^{*}$ is the source antenna temperature corrected for atmospheric attenuation and all telescope losses (ohmic and spillover) except for coupling of the source and beam. We detected $\mathrm{DCN}$ at $4 / 5$ additional positions offset by $1^{\prime}$ and $2^{\prime} \mathrm{S}$ from our center position and also observed the $\mathrm{J}=1-0$ lines of $\mathrm{DNC}, \mathrm{HC}^{15} \mathrm{~N}, \mathrm{H}^{13} \mathrm{CN}$, $\mathrm{HCN}, \mathrm{HCO}^{+}$, and HNC. As a check we observed the known DCN lines in the Sgr B2 molecular at the $(\mathrm{OH})$ position $\left(\alpha=17^{h} 44^{m} 11^{s} ; \delta=-28^{\circ} 22^{\prime} 30^{\prime \prime}\right)$ where the DCN 1-0 line is blended with the $\mathrm{H}_{2} \mathrm{CO} 5(1,4)-5(1,5)$ transition.

The spectra are shown in figures 1 and 2 and our results are given in tables 1, 2, and 3 where the lines were identified from the Lovas (1992) rest frequencies (unknown lines listed with $\mathrm{U}$ ) and fit by Gaussian profiles.

Table 1. $\quad$ Sgr A $50 \mathrm{~km} / \mathrm{s}$ Cloud, Center Position

\begin{tabular}{lrrrrr}
\hline Molecule & Transition & $\nu \mathrm{MHz})$ & $T_{r}^{*}(\mathrm{mK})$ & $\mathrm{rms}(\mathrm{mK})$ & $\Delta \mathrm{V}(\mathrm{km} / \mathrm{s})$ \\
\hline & & $\mathrm{U} 72323.9$ & 68 & 7.1 & 22.7 \\
& & $\mathrm{U} 72344.2$ & 18 & 7.1 & 30.6 \\
$\mathrm{DCN}$ & $1-0$ & 72414.9 & 61 & 7.1 & 30.9 \\
$\mathrm{HC}^{13} \mathrm{CCN}$ & $8-7$ & 72475.1 & 145 & 7.1 & 21.9 \\
$\mathrm{HCC}^{13} \mathrm{CN}$ & $8-7$ & 72482.1 & 120 & 7.1 & 27.9 \\
$\mathrm{HC}^{15} \mathrm{~N}$ & $1-0$ & 86055.0 & 192 & 27 & 26.8 \\
$\mathrm{SO}$ & $2(2)-1(1)$ & 86093.5 & 226 & 27 & 19.8 \\
$\mathrm{H}^{13} \mathrm{CN}$ & $1-0$ & 86340.2 & 1510 & 20 & 35.2 \\
$\mathrm{HCN}$ & $1-0$ & 88631.8 & 5408 & 48 & 27.1 \\
$\mathrm{HCO}^{+}$ & & 89188.5 & 3070 & 23 & 25.7 \\
& & $\mathrm{U} 89204.3$ & 532 & 23 & 11.7 \\
& & $\mathrm{U} 89215.5$ & 250 & 23 & 11.7 \\
$\mathrm{HC}^{13} \mathrm{CCN}$ & & $\mathrm{U} 89221.8$ & 130 & 23 & 7.8 \\
$\mathrm{HCC}{ }^{13} \mathrm{CN}$ & $10-9$ & 90593.1 & 130 & 23 & 18.5 \\
$\mathrm{HNC}$ & $10-9$ & 90601.8 & 120 & 23 & 25 \\
$\mathrm{C}_{2} \mathrm{~S}$ & $1-0$ & 90663.5 & 2198 & 23 & 41.3 \\
& $7,7-6,6$ & 90686.4 & 167 & 23 & 12.4 \\
$\mathrm{DCN}$ & & $\mathrm{U} 144735.3$ & 67.6 & 15 & 12.6 \\
& $2-1$ & 144828.0 & 38.9 & 15 & 25.2 \\
\hline
\end{tabular}

We used 1-MHz filters; a $256 \mathrm{MHz}$ bandwidth; $4.14 \mathrm{~km} / \mathrm{s}$ resolution and 86 " beam at $72 \mathrm{GHz}, 2.07 \mathrm{~km} / \mathrm{s}$ resolution and 43 " beam at $144 \mathrm{GHz}$. Pointing 

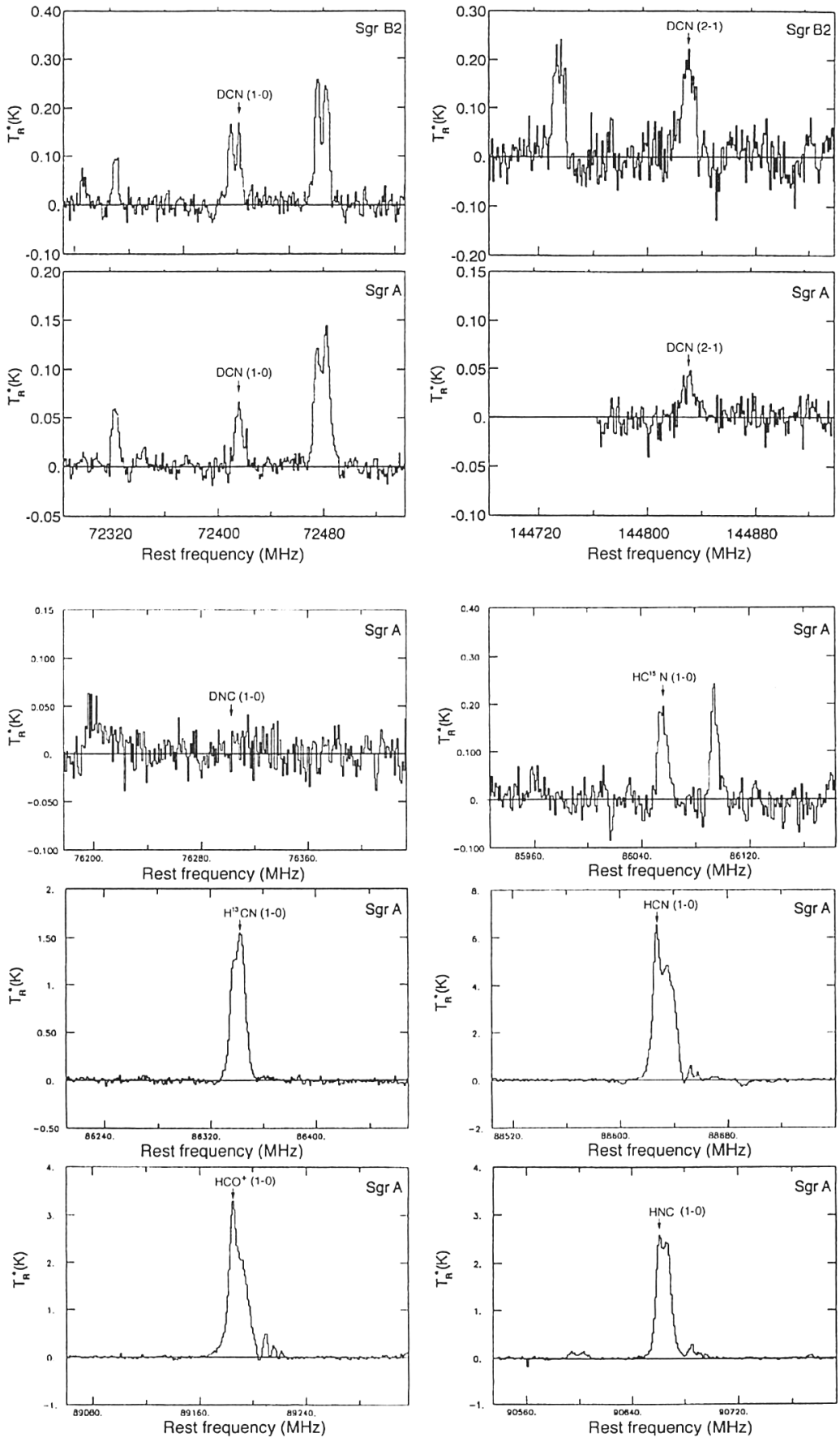

Figure 1. Spectra of the GC Sgr A $50 \mathrm{~km} / \mathrm{s}$ and Sgr B2 molecular clouds. The $\mathrm{J}=1-0$ lines of $\mathrm{DCN}, \mathrm{DNC}, \mathrm{HC}^{15} \mathrm{~N}, \mathrm{HC}^{13} \mathrm{~N}, \mathrm{HCN}, \mathrm{HCO}^{+}$, and HNC are labeled. 
Table 2. $\quad$ Sgr A $50 \mathrm{~km} / \mathrm{s}$ Cloud, Offset Positions

\begin{tabular}{|c|c|c|c|c|c|}
\hline Molecule & Transition & $\nu \mathrm{MHz})$ & $T_{r}^{*}(\mathrm{mK})$ & $\mathrm{rms}(\mathrm{mK})$ & $\Delta \mathrm{V}(\mathrm{km} / \mathrm{s})$ \\
\hline \multicolumn{6}{|l|}{ 1'West } \\
\hline & & U72324.9 & 31 & 16 & 25.2 \\
\hline $\mathrm{DCN}$ & $1-0$ & 72414.9 & 30 & 16 & 41.5 \\
\hline $\mathrm{HC}^{13} \mathrm{CCN}$ & $8-7$ & 72475.1 & 102 & 16 & 21.4 \\
\hline $\mathrm{HCC}^{13} \mathrm{CN}$ & $8-7$ & 72482.1 & 91 & 16 & 21.4 \\
\hline \multicolumn{6}{|l|}{ 1'North } \\
\hline & & U72323.1 & 23 & 14 & 62.9 \\
\hline $\mathrm{DCN}$ & $1-0$ & 72414.9 & 11 & 14 & 59.1 \\
\hline $\mathrm{HC}^{13} \mathrm{CCN}$ & $8-7$ & 72475.1 & 60 & 14 & 15.1 \\
\hline $\mathrm{HCC}^{13} \mathrm{CN}$ & $8-7$ & 72482.2 & 58 & 14 & 22.8 \\
\hline \multicolumn{6}{|l|}{ 1' East } \\
\hline DCN & $1-0$ & 72419.0 & 40 & 19 & 33.1 \\
\hline $\mathrm{HC}^{13} \mathrm{CCN}$ & $8-7$ & 72475.1 & 100 & 19 & 27.3 \\
\hline $\mathrm{HCC}^{13} \mathrm{CN}$ & $8-7$ & 72482.1 & 100 & 19 & 27.3 \\
\hline \multicolumn{6}{|l|}{ 1'South } \\
\hline & & U72324.5 & 64 & 16 & 19.7 \\
\hline DCN & $1-0$ & 72414.9 & 55 & 16 & 33.9 \\
\hline $\mathrm{HC}^{13} \mathrm{CCN}$ & $8-7$ & 72475.1 & 135 & 16 & 18.8 \\
\hline $\mathrm{HCC}^{13} \mathrm{CN}$ & $8-7$ & 72482.1 & 118 & 16 & 20.1 \\
\hline \multicolumn{6}{|l|}{ 2' South } \\
\hline DCN & $1-0$ & 72414.9 & 32 & 15 & 27.1 \\
\hline $\mathrm{HC}^{13} \mathrm{CCN}$ & $8-7$ & 72475.1 & 80 & 15 & 18.6 \\
\hline $\mathrm{HCC}^{13} \mathrm{CN}$ & $8-7$ & 72482.1 & 79 & 15 & 27.5 \\
\hline
\end{tabular}

Table 3. $\quad$ Sgr B2 $(\mathrm{OH})$

\begin{tabular}{lrrrrr}
\hline Molecule & Transition & $\nu \mathrm{MHz})$ & $T_{r}^{*}(\mathrm{mK})$ & $\mathrm{rms}(\mathrm{mK})$ & $\Delta \mathrm{V}(\mathrm{km} / \mathrm{s})$ \\
\hline $\mathrm{CH}^{13} \mathrm{CCH}_{3}$ & $10(1,9)-10(0,10)$ & 72300.2 & 61 & 16 & 18.1 \\
& & $\mathrm{U} 72324.0$ & 106 & 16 & 17.3 \\
$\mathrm{H}_{2} \mathrm{CO}$ & $5(1,4)-5(1,5)$ & 72404.4 & 167 & 16 & 18.6 \\
$\mathrm{DCN}$ & $1-0$ & 72414.9 & 167 & 16 & 16.6 \\
$\mathrm{HC}^{13} \mathrm{CCN}$ & $8-7$ & 72475.1 & 226 & 16 & 20.0 \\
$\mathrm{HCC}^{13} \mathrm{CN}$ & $8-7$ & 72482.1 & 260 & 16 & 20.0 \\
& & $\mathrm{U} 144734.8$ & 217 & 38 & 16.9 \\
$\mathrm{DCN}$ & $2-1$ & $\mathrm{U} 144828.0$ & 196 & 38 & 21.5 \\
\hline \hline
\end{tabular}


was checked using Jupiter and Uranus. The double sideband $T_{\text {sys }}$ was $350 \mathrm{~K}$ at $72 \mathrm{GHz}$ and $400 \mathrm{~K}$ at $144 \mathrm{GHz}$. We could not resolve the hyperfine splitting for the $\mathrm{DCN}, \mathrm{HCN}, \mathrm{H}^{13} \mathrm{CN}$, and $\mathrm{HC}^{15} \mathrm{~N}$ lines because of our use of $1 \mathrm{MHz}$ filters and the large line widths of $\approx 30 \mathrm{~km} / \mathrm{s}$.

Because the HCN $\mathrm{J}=1-0$ line is optically thick, we used the optically thin $\mathrm{J}=1-0$ line of $\mathrm{HC}^{15} \mathrm{~N}$ to estimate DCN $/ \mathrm{HC}^{15} \mathrm{~N}$ and DCN/HCN (Hatchell, Millar \& Rodgers 1998). DCN $/ \mathrm{HCN}=T_{D C N} \Delta \mathrm{V} / T_{H C^{15} N} \Delta \mathrm{V}\left({ }^{15} N /{ }^{14} N\right)_{e^{-}}\left(\Delta \mathrm{E} / \mathrm{k} T_{e x}\right)$, where $\left(\Delta \mathrm{E}\right.$ is difference between the energies of $\mathrm{DCN}$ and $\mathrm{HC}^{15} \mathrm{~N}$ transitions, $T_{e x}=75 \mathrm{~K}$, and sources are extended relative to our beam for the $\mathrm{J}=1$ 0 lines. We obtained $\mathrm{DCN} / \mathrm{HC}^{15} \mathrm{~N}=0.36$ and $\mathrm{DCN} / \mathrm{HCN}=4.0 \times 10^{-4} \mathrm{using}$ $\left({ }^{15} N /{ }^{14} N\right)=900$ (Güsten \& Ungerechts 1985) which are the lowest DCN $/ \mathrm{HC}{ }^{15} \mathrm{~N}$ and $\mathrm{DCN} / \mathrm{HCN}$ ratios observed in any molecular cloud.

\section{Discussion}

Chemical fractionation resulting from the lower zero-point energy for deuterated molecules due to its larger mass significantly increases these abundances. Our chemical model is an updated version of Rodgers \& Millar (1996) containing 165 non-deuterated, 122 deuterated species, 5192 gas-phase reactions, recent data on the dissociative recombination of $\mathrm{H}_{2} D^{+}, \mathrm{S}$ chemistry, and grain formation of $\mathrm{H}_{2}$ and $\mathrm{HD}$. For a model with the physical parameters typical of the 50 $\mathrm{km} / \mathrm{s}$ cloud $\left(\mathrm{T}=75 \mathrm{~K} ; \mathrm{n}(\mathrm{H})=10^{6} \mathrm{~cm}-3\right.$; an ionization rate of $1.3 \times 10^{17} \mathrm{sec}^{-1}$; fractional abundances of $\mathrm{C}, \mathrm{N}$, O equal to $9.9 \times 10^{-4}, 3.6 \times 10^{-4}$, and $1.98 \times 10^{-3}$, respectively, approximately $3 \times$ their solar abundances; Minh, Irving, \& Friberg (1992); Serabyn, Lacy, \& Actermann (1992); Poglitsch et al. (1991),Simpson et al. (1995)), we obtained a best agreement with the observed DCN/HCN for $\mathrm{D} / \mathrm{H}=3 \times 10^{-6}$ (a degree of fractionation of 133 ).

Although the column densities of DCN and HCN will be changed by varying the physical conditions, the fractionation is independent of density, metallicity, and ionization rate where a faster ionization rate resulted a shorter time to reach the steady-state values. $\mathrm{DCN} / \mathrm{HCN}$ as a function of $\mathrm{T}$ and $\mathrm{n}$ is shown in figure 3 . Our $\mathrm{D} / \mathrm{H}$ is also consistent with the upper limits of $\mathrm{D} / \mathrm{H}<8.3 \times 10^{-5}$ (from the 92-cm hfs line; Lubowich, Anantharamaiah, \& Pasachoff 1989)and DCN/HCN $<6 \times 10^{-4}$ (Jacq et al. 1999)in GC $50 \mathrm{~km} / \mathrm{s}$ molecular cloud. There is one reported marginal $1 \sigma$ detection of $\mathrm{D}$ from the $\mathrm{J}=1-0$ line of $\mathrm{DCN}$ in the 50 $\mathrm{km} / \mathrm{s}$ Sgr A molecular cloud core (Penzias 1979) with $\mathrm{T}_{a}^{*}=0.02 \pm 0.015 \mathrm{~K}$.

Using a closed-box model and a time scale for GC astration of $2 \times 10^{8} \mathrm{yr}$ Audouze et al. (1976) calculate $\mathrm{D} / \mathrm{H}=5 \times 10^{-12}$. If there were no additional sources of D, the GC molecular clouds should be composed of astrated material completely depleted in D and DCN should not be detectable. If D is produced via any mechanism related to the nucleosynthesis of $\mathrm{O}$ (massive stars or type-II $\mathrm{SN}$ ), then the $\mathrm{D}$ and $\mathrm{O}$ abundances will be positively correlated. Because the GC O abundance is enhanced by $3 \times$ while the $\mathrm{D}$ abundance is reduced by $4.5 \times$, $\mathrm{D}$ is anticorrelated with the $\mathrm{O}$ abundance. Thus if any $\mathrm{D}$ nucleosynthesis exists, it is not correlated with $\mathrm{O}$ nucleosynthesis or massive stars.

Combining our result of the GC D/H $=3.0 \times 10^{-6}$, the Sgr B2(OH) D/H = $5.0 \times 10^{-6}$ (Jacq et al. 1999), the local ISM D/H $=1.5 \times 10^{-5}$ (Linsky 1998), and the possible detection of DI with $\mathrm{D} / \mathrm{H}=3.9 \times 10^{-4}$ in the Galactic anticenter 




Figure 2. Spectra of the GC $50 \mathrm{~km} / \mathrm{s}$ molecular cloud offset by $1^{\prime}$ North, South, East, West and by $2^{\prime}$ South of our center position. 
$[\mathrm{DCN}] /[\mathrm{HCN}]$

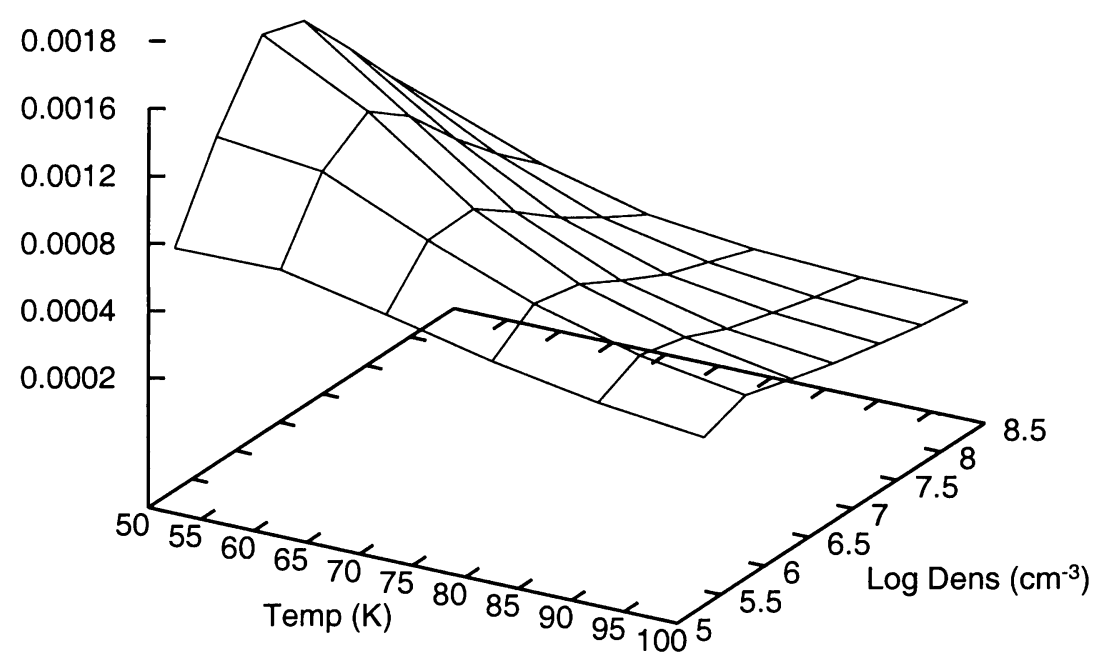

Figure 3. $\mathrm{DCN} / \mathrm{HCN}$ as a function of temperature of temperature and density

(Chengalur, Braun, \& Burton, 1997), we obtain a positive abundance gradient of $\mathrm{D}$ in the Galaxy indicating that there is no significant Galactic production of D (Pasachoff \& Vidal-Madjar 1988). The most likely source of the GC D is continuous infall of primordial matter with $\mathrm{D} / \mathrm{H} \approx 5 \times 10^{-5}$ where the resultant $\mathrm{D} / \mathrm{H}$ ratio is determined by the astration and mixing which always reduces the $\mathrm{D} / \mathrm{H}$. This replenishment would negate much of the effects of astration and inject gas enhanced in $\mathrm{D}$ but deficient in $\mathrm{O}$ gas into the GC. This is in agreement with the analysis of the Galactic D/O ratio (Prantzos 1996) where the ISM D is probably the result of infall plus astration.

Our results constrain models of $\mathrm{GC}$ activity that predict a $\mathrm{GC} \mathrm{D} / \mathrm{H}=$ $10^{-4}$ (10 pc from the GC) from $\gamma$-ray photospallation reactions (Boyd, Ferland, \& Schramm 1989) for an AGN or Seyfert $\gamma$-ray luminosity $L_{\gamma}=10^{42} \mathrm{erg} / \mathrm{s}$; $\mathrm{D} / \mathrm{H}=10^{-2}$ and $L_{\gamma}=10^{44} \mathrm{erg} / \mathrm{s}$ for a quasar; and $\mathrm{D} / \mathrm{H}=2 \times 10^{-5}$ for an AGN cosmic-ray (CR) proton luminosity $L_{p}=10^{43} \mathrm{erg} / \mathrm{s}$ for 1 Gyr (Ozernoi \& Chernomordik 1975). The GC production of D by large fluxes of CRs or $\gamma$-rays in the early Galaxy is possible only if there was rapid astration so that any $\mathrm{D}$ produced was destroyed in the GC but there was little astration in the local ISM. Because the current GC D/H $<<\mathrm{D} / \mathrm{H}$ predicted by quasar or AGN activity, there has not been recent AGN activity in the GC. The luminosities required to produce the $\mathrm{GC} \mathrm{D} / \mathrm{H}$ are still many orders of magnitude larger than the observed GC $L \gamma$ or $L_{p}=2 \times 10^{37} \mathrm{erg} / \mathrm{s}$ (Mayer-Hasselwander et al. 1998; Mastichades \& Ozernoy 1994). The $\gamma$-rays from CR spallation reactions were not detected during 9 years of GC observations (Harris, Share, \& Messina, 1995).

Thus AGN activity, ( $\gamma$-ray photodisintegration reactions, or CR spallation reactions are not significant sources of Galactic deuterium. Because almost all nucleosynthesis processes that can produce a significant abundance of $\mathrm{D}$ always 
overproduce $\mathrm{Li}$ or $\mathrm{B}$ by $10^{3}-10^{5}$ times, the observed upper limit on the GC $\mathrm{Li}$ of $(\mathrm{Li} / \mathrm{H})_{G C}<3.9 \times 10^{-8}$ or $(\mathrm{Li} / \mathrm{H})_{G C}<20(\mathrm{Li} / \mathrm{H})_{\text {disk }}$, (Lubowich, Turner, \& Hobbs 1998) further implies that there are no Galactic Center sources of deuterium. Although weak AGN activity or periodic bursts of star formation may occur, the Milky Way has not had a recent active phase nor an early active phase for any period longer than 1 Gyr. Although unlikely, we cannot exclude low astration models with no infall or a continuous production of deuterium combined with the exact astration necessary to produce the Galactic Center, the local, and the anticenter $\mathrm{D} / \mathrm{H}$ values.

If all the deuterium is primordial and the astration models Prantzos (1996) are correct, then the primordial or early Galactic $\mathrm{D} / \mathrm{H} \approx 5 \times 10^{-5}$. For this $\mathrm{D} / \mathrm{H}$ big-bang nucleosynthesis models imply that the baryon-to-photon ratio ratio $\eta_{b}=3 \times 10^{-10}$, there are less than four neutrino families, the baryon density of the Universe $\rho_{b}=3 \times 10^{-31} \mathrm{gm} \mathrm{cm}^{-3}<$ critical density of $\rho_{c}=3 H_{o}^{2} / 8 \pi G=$ $1.88 H_{o}^{2} \times 10^{-29} \mathrm{gm} \mathrm{cm}^{-3}=9.2 \times 10^{-30} \mathrm{gm} \mathrm{cm}^{-3}$ (for a Hubble constant $H_{o}$ $=70 \mathrm{~km} / \mathrm{s} / \mathrm{Mpc}$ ) necessary to close the Universe for a flat Einstein-de Sitter Universe, and $\Omega_{b}=\rho_{b} / \rho_{c}=0.04$ (Copi, Schramm, \& Turner, 1995). Thus the fraction of the critical density contributed by baryons $\left(\Omega_{b}\right)$ in a closed Universe requires that most of the baryons are in the form of dark matter.

Note added in proof: Based on additional modelling $\mathrm{D} / \mathrm{H}=1.7 \times 10^{-6}, 9 \times$ lower than the local $\mathrm{D} / \mathrm{H}$ which strengthens our conclusion that $\mathrm{GC} \mathrm{D}$ is the result of infalling matter.

We thank Ann Mancuso (Hofstra) Sebastian Diaz (Williams), Matthew Pickard (Keck Northeast Astronomy Consortium Summer Fellow), Ken Pagliuca (AIP), \& M. L. Kutner. We acknowledge a Hofstra Faculty Research and Development Grant, a Bronfman Science Center Grant from Williams, and a PPARC grant at UMIST.

\section{References}

Audouze, J., Lequeux, J., Reeves, H., \& Vigroux, L. 1976, ApJ, 208, L51

Boyd, R.N., Ferland, G.J., \& Schramm, D.N. 1989, ApJ, 336, L1

Chengalur, J. N., Braun, R., \& Butler, Burton W. 1997, A\&A, 318, L35.

Copi, C. J., Schramm, D.N, \& Turner, M.S. 1995, Phys. Rev. Letters, 75, 3981

Genzel, R., Hollenbach, D., \& Townes, C.H. 1994, Reports Prog. Phys., 57, 417

Güsten, R. \& Ungerechts, H. 1985, A\&A, 145, 241

Harris, Michael J., Share, Gerald H., \& Messina, Daniel C. 1995, ApJ, 448, 157

Hatchell, J. , Millar, T.J., \& Rodgers, S. D. 1998, A\&A, 332, 695.

Jacq, T., Baudry, A., Walmsley, C.M., \& Caselli, P., 1999, A\&A, 347, 957

Linsky, J. L. 1998, Space Sci. Rev. 84, 285

Lovas, F. J. 1992. J. Phys. \& Chem. Reference Data 21, 181

Lubowich, D. A., Anantharamaiah, K.R., \& Pasachoff, J.M. 1989, ApJ, 345, 770

Lubowich, D. A., Turner, B.E., \& Hobbs, L.M. 1998 ApJ, 508, 729

Mastichades, A. \& Ozernoy, L. M. 1994, ApJ, 426, 599

Mayer-Hasselwander et al. 1998, A\&A, 335, 161 
Minh, Y.C., Irvine, W.M., \& Friberg, P. 1992, A\&A, 258, 489

Ozernoi, L., \& Chernomordik, V.V. 1975, Sov. Astron. 19, 693

Pasachoff, J. M, \& Vidal Madjar, A. 1989, Comments in Astrophysics, 14, 61

Penzias, A.A. 1979, ApJ, 228, 430

Poglitsch, A., Stacey, G. J., Geis, N., Haggerty, M., Jackson, J., Rumitz, M., Genzel, R., Townes, C. H. 1991, ApJ, L33

Prantzos, N. 1996, A\&A, 310, 106

Rodgers, S.D. \& Millar, T.J. 1996, MNRAS, 280, 1046

Schramm, D. N. , \& Turner, M. S. 1998, Rev. Mod. Physics, 70, 303

Serabyn, E., Lacy, J.H., \& Actermann, J.M. 1992, ApJ395, 166

Simpson, J.P., Colgan, S.W.J., Rubin, R.H., Erickson, E.F., \& Haas, M.R. 1995, ApJ, 444, 721

Vidal-Madjar, A. 2000, Nucl. Phys. B (Proc. Suppl.), 80119 